\title{
Author Correction: The regulation, functions and clinical relevance of arginine methylation
}

Ernesto Guccione and Stéphane Richard (iD

Nature Reviews Molecular Cell Biology https://doi.org/10.1038/s41580-019-0155-x Published online 26 July 2019

In Table 1, the $\mathrm{IC}_{50}$ values of SGC3027 should be $2.5 \mathrm{nM}$ in vitro and $2.4 \mu \mathrm{M}$ in cells, and not as previously published. The mistake has been corrected in the HTML and PDF versions of the article.

https://doi.org/10.1038/s41580-019-0166-7 I Published online 2 August 2019 